\title{
A COMPARISON BETWEEN STRUCTURE-FROM-MOTION AND TERRESTRIAL LASER SCANNING FOR DERIVING SURFACE ROUGHNESS: A CASE STUDY ON A SANDY TERRAIN SURFACE
}

\author{
L. Fan ${ }^{1 *}$ \\ ${ }^{1}$ Department of Civil Engineering, Xi' an Jiaotong - Liverpool University, Suzhou, P.R. China - Lei.Fan@xjtlu.edu.cn
}

KEY WORDS: Point Clouds, Surface Roughness, Structure-From-Motion, Terrestrial Laser Scanning

\begin{abstract}
:
Structure-from-motion (SfM) is a useful technique for acquiring the topographic information of terrain surfaces for a wide range of geoscience applications. Due to its easy mobilization and cost-effective implementation, the SfM technique may be considered as a favourable alternative to the laser scanning technique in some applications. To this end, it is essential to understand how point cloud data derived using these two different surveying techniques affect the geographic information system (GIS) outputs such as local surface roughness of a terrain surface. In this case study, a small sandy terrain surface was surveyed using a terrestrial laser scanner and the digital camera of a mobile phone, respectively. Analyses were carried out to check the measurement quality of the SfMderived point cloud and to explore the differences in local surface roughness calculated using the SfM-derived point cloud and that from the scanner, respectively. In addition, it looked into how those differences were affected by different surface roughness descriptors and the associated input parameters (mainly window sizes). Two commonly used methods for describing local surface roughness were considered, consisting of root mean square height and standard deviation of slope.
\end{abstract}

\section{INTRODUCTION}

Point cloud data are now used widely for various geographic information system (GIS) tasks, for example, construction of fine-resolution digital elevation models, calculation of GIS parameters (e.g. surface roughness) and measurement of terrain surface movements (Chen and Zhou, 2013; Fan et al., 2014; Brunori, et al., 2013). In geoscience, there are mainly two types of techniques for acquiring point cloud data: laser scanning systems such as terrestrial laser scanning (TLS) and airborne light detection and ranging (LiDAR), and imagery-based photogrammetric techniques such as structure-from-motion (SfM). In recent years, there are an increasing number of applications of the SfM technique for deriving point clouds for various geoscience applications (Westoby, 2012; Colomina, 2014; Prosdocimi et al., 2015; Smith et al., 2016; Marteau et al.; 2017). In this technique, a set of images of a scene are used to automatically solve the geometry of the scene, camera positions and orientations without the need to specify a priori, a network of targets of known positions (Westoby, 2012). A hand-held digital camera (e.g. a mobile phone) or a compact digital camera mounted on a light-weight drone can be used for acquiring images. These offer an attractive surveying approach that has various advantages, for example, low cost and easy mobilization of field surveying as compared to laser scanning techniques.

Terrain surface roughness is an important parameter for describing terrain surface variability or complexity in Earth science. It is often used for investigating the DEM error [Aguilar et al., 2005; Kraus et al. 2006; Fan and Atkinson, 2015, 2018] and for studying Earth surface processes and landforms [Nield and Wiggs, 2011; Grohmann et al. 2011; Milenković et al., 2015]. There exist a wide range of methods for estimating surface roughness. For point cloud data representing a terrain surface, the method most commonly used for estimating terrain surface roughness is root mean square height (RMSH) [Nield and Wiggs, 2011; Hugenholtz et al., 2013; Brubaker et al. 2013]. Another popular method is standard deviation of slope, which was found to be a very effective measure of surface roughness by Grohmann et al. (2011). These two methods are considered in this study.

Based on the aforementioned, the SfM technique may be considered as a more cost-effective and convenient alternative to laser scanners for obtaining point clouds for characterising local terrain surface roughness. As the characteristics of measurement errors in SfM-derived point clouds are unlikely to be the same as those obtained using TLS, it is important to understand the likely similarities or differences in surface roughness derived using those two techniques. However, there are few reported case studies in the literature for comparing surface roughness calculated using SfM-derived and TLSderived point clouds. As such, the main purpose of this study is to explore the likely differences using a case study where a small sandy terrain surface is considered.

\section{METHODOLOGY}

\subsection{Study site and data}

For ease of access, a man-made sandy area shown in Figure 1a on the campus was used for the experiment. The size of the sandy area was approximately $10 \mathrm{~m} 2$ and a sub-area of it was selected for a detailed study. The sandy terrain surface was first surveyed using a Leica ScanStation P40 scanner from three stations. Subsequently, an iPhone 7 was used to take a total number of 36 images from multiple locations and orientations. Terrestrial laser scanning (TLS) is an accurate survey technique, which was used to assess the measurement accuracy of the point clouds derived from images. To enable data registration into the

\footnotetext{
* Corresponding author
} 
same coordinate system, three Leica targets placed on the ground surface were measured using the scanner and imaged using the camera of the iPhone, respectively. As the laser scanning data and those derived from the images need to be compared, the registration error should be minimised. As such, the targets were arranged to encircle the sub-area of interest but were close enough to the sub-area considered so that those target centres could be identified more accurately in SfMderived point clouds. This is the main reason why only a subarea (instead of the whole area) was selected for detailed analyses. The sub-area, the size of which is approximately $1 \mathrm{~m}$ by $1 \mathrm{~m}$, is highlighted by the dashed line in Figure 1a. Several small plates were placed on the terrain surface for determining the measurement precision.

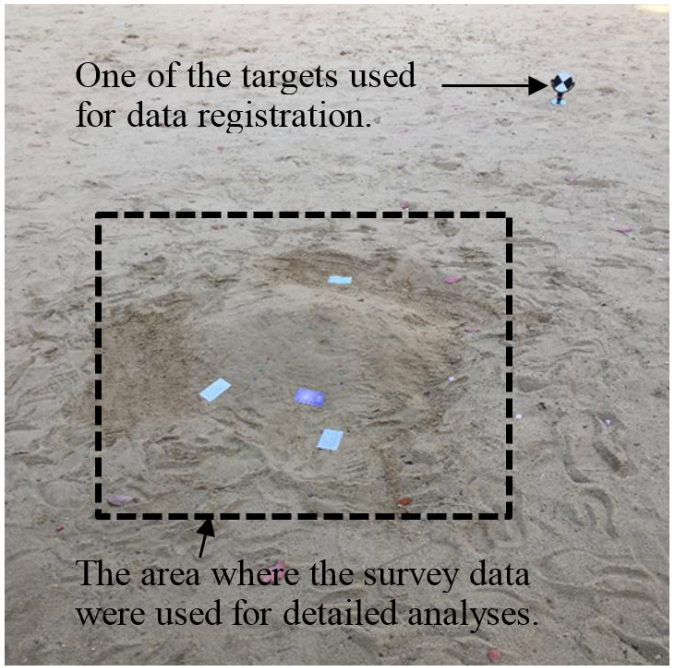

(a)

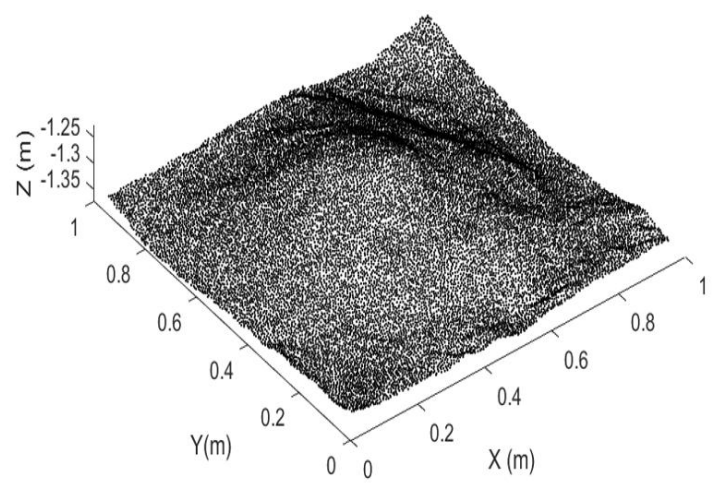

(b)

Figure 1. (a) The study site; (b) The SfM-derived point cloud.

The point cloud obtained using the scanner for the sub-area consisted of approximately 22000 data points (referred to as TLS-whole), which is equivalent to an average data spacing of approximately $6.8 \mathrm{~mm}$. The densities of the point clouds derived from images varied with the quality settings used in the data processing. The point cloud (referred to as Photo-whole) derived from images using highest quality settings is shown in Figure $1 \mathrm{~b}$, which is a thinned point cloud (using the minimum distance method) of the original one to match the density of the TLS data. Based on the TLS-whole point cloud, two subset point clouds were generated: TLS-subset1 and TLS-subset2. These two subset point clouds did not share the same data points, and each had approximately 8000 data points (equivalent to an average data spacing of approximately $11 \mathrm{~mm}$ ). Similarly, two subset point clouds (Photo-subset1 and Photo-subset2) of approximately 8000 data points were created from the Photowhole. The purpose of generating these subsets was to observe the differences in the surface roughness maps derived if the same source of data (thus similar measurement errors) was used.

\subsection{Data analysis}

The imagery data were processed in Agisoft PhotoScan to generate point clouds. For generating sparse and dense point clouds, there were various quality options, including lowest, low, medium, high and highest/ultrahigh. As these options would affect measurement errors in the SfM-derived point clouds, these quality settings were tested first. The positional information of the targets obtained by the scanner was used to validate and adjust the scale of the SfM-derived point clouds.

For registering the TLS point clouds acquired at three different stations, only the targets were used. This process was implemented in Leica Cyclone. To register the SfM-derived point clouds to the TLS point cloud, two steps were adopted in CloudCompare. The coordinates of the target centres were extracted and used for initial registration, which was followed by a cloud-to-cloud registration (i.e. the ICP algorithm) that led to a $100 \%$ overlap theoretically.

The point clouds representing the small plates were cropped from raw data. A plane was fitted to each set of the data points cropped and the residuals from the fitted plane were calculated to check the measurement precision of the SfM-derived point clouds at various quality settings. In addition, the SfM-derived point cloud using the highest quality setting was compared to the TLS point cloud by interpolation to a set of regular grid locations of $0.01 \mathrm{~m}$ by $0.01 \mathrm{~m}$ resolution. This was used to assess the measurement accuracy of the SfM-derived point cloud.

Two surface roughness methods were considered, consisting RMSH and standard deviation of slope. The RMSH values were calculated at some pre-defined grid locations with a spatial resolution of $0.01 \mathrm{~m}$ by $0.01 \mathrm{~m}$. A local square window centred at each grid location was used to select the neighbouring data points surrounded by the window, and the data points selected were used to calculate the local RMSH value. For the subset point clouds (i.e. TLS-subset1, TLS-subset2, Photo-subset1 and Photo-subset2) considered, the moving window sizes considered included $3,5,7,9,11$ and 13 times their average data spacing (i.e. equivalent to window sizes of $33 \mathrm{~mm}, 55 \mathrm{~mm}$, $77 \mathrm{~mm}, 99 \mathrm{~mm}, 121 \mathrm{~mm}$ and $143 \mathrm{~mm}$, respectively). For the TLS-whole and the Photo-whole point clouds, the same window sizes as those used for the subset point clouds were adopted, which were equivalent to approximately $5,8.3,11.7,15,18.5$, 21.5 times the average data spacing of the TLS-whole or the Photo-whole point clouds. The two surface roughness methods were applied to the six aforementioned datasets.

\section{RESULTS AND DISCUSSION}

Figure 2 shows the measurement precision of the SfM-derived point clouds that represent the plate used. It is observed that the quality choices for point cloud densification had a more significant effect on the precision. The improvement in the precision using a higher quality setting for generating sparse point clouds was comparatively small. The results suggested that there was little justification for using the highest/ultrahigh quality setting for generating sparse and dense point clouds. It is 
not only because of the limited improvement but also because the computational time increased exponentially with those quality settings. However, one advantage of using the highest/ultrahigh quality is that the density of point cloud data derived was very high, which may be useful in certain applications (e.g. in this study, fine-resolution point cloud data were required for more accurately identifying the target centres for registration).

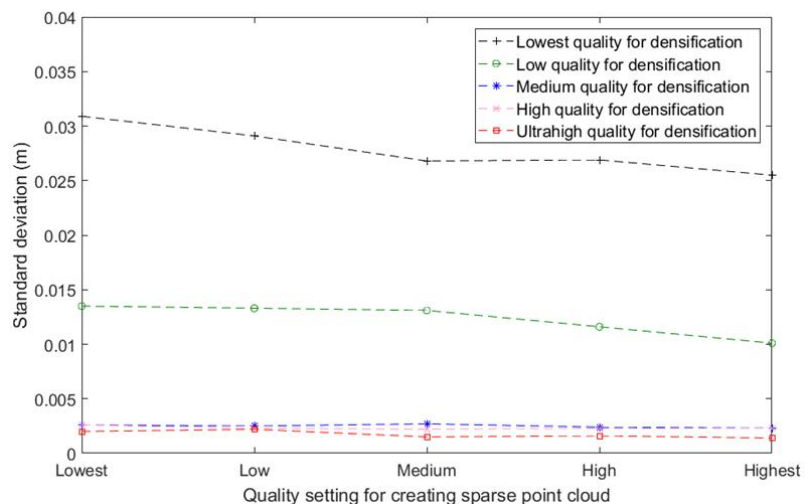

Figure 2. The precision of the SfM-derived point clouds at different quality settings
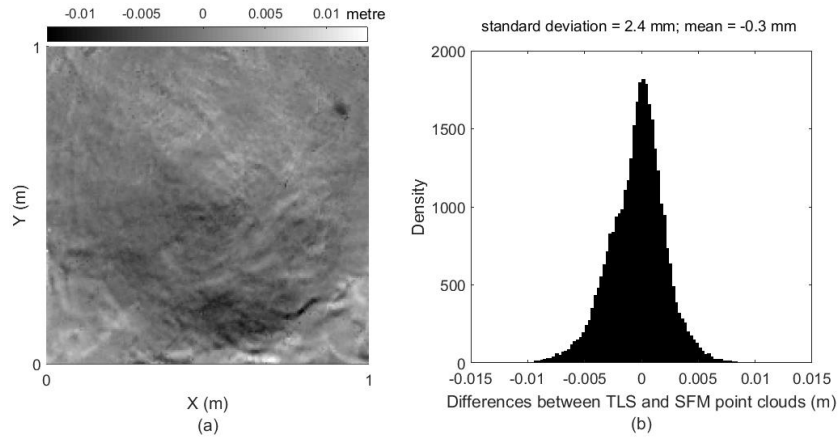

Figure 3. The accuracy of the SfM-derived point cloud.

For the sub-area investigated, the SfM-derived point cloud (using the highest quality setting) was compared to the TLS point cloud (i.e. the reference data). Their differences in elevations are shown in Figure 3 and represent the measurement accuracy of the SfM-derived point cloud. Figure 3a shows that the differences were spatially auto-correlated, suggesting the likely auto-correlation of measurement errors in the SfMderived point cloud (assuming that the measurement errors in the TLS point cloud were random spatially). However, it is also possible that the spatial auto-correlation was caused partly by the imperfect registration between the two datasets. The standard deviation of the elevation differences was approximately $2.4 \mathrm{~mm}$, suggesting that the accuracy of the SfM-derived point cloud was high. In addition, there was effectively no bias as the mean difference was very small (i.e. $-0.3 \mathrm{~mm}$ ). The aforementioned results show that the quality of the SfM-derived point cloud was close to the TLS point cloud. However, the characteristics of the measurement errors in these two types of datasets might be different. Consequently, the local surface roughness calculated using these datasets were likely to vary because the measurement errors can change the local spatial variation and thus affect local surface roughness.
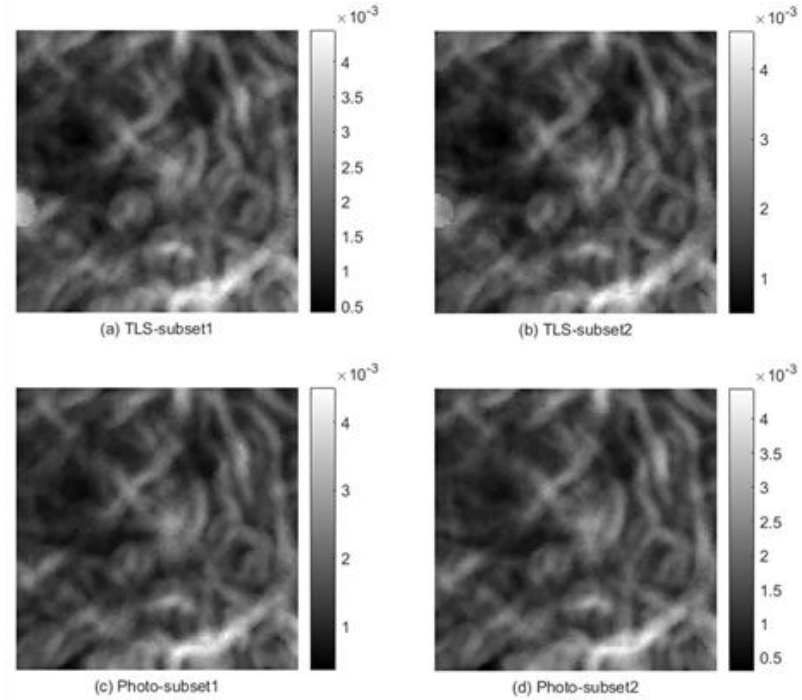

Figure 4. The RMSH for the window size of $76 \mathrm{~mm}$ (i.e. 7 times the average data spacing).

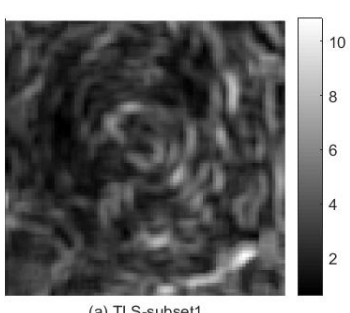

(a) TLS-subset1

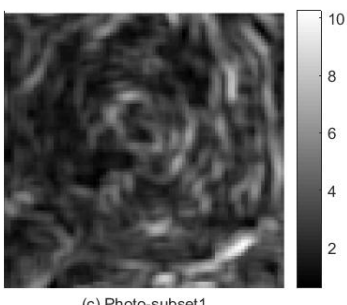

(c) Photo-subset1

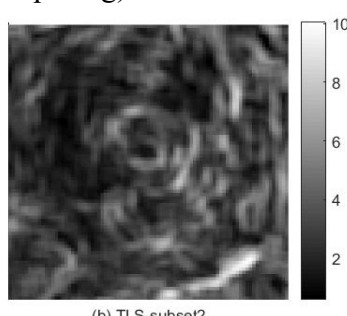

(b) TLS-subset2

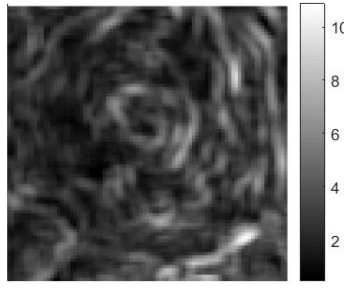

(d) Photo-subset2
Figure 5. The standard deviation of slope for the window size of $76 \mathrm{~mm}$ (i.e. 7 times the average data spacing).

The surface roughness maps calculated using the RMSH and the standard deviation of slope are shown in Figure 4 and Figure 5, respectively. Only those constructed using the subset point clouds and a window size of $77 \mathrm{~mm}$ are shown. To enable a more clear comparison between the surface roughness maps derived using each dataset, the coefficient of determination R2 was calculated for each pair of roughness maps under comparison. The results were summarised graphically in Figure 6. When the same source of point cloud data was considered (i.e. TLS-subset1 v.s. TLS-subset 2 or Photo-subset1 v.s. Photosubset2), the correlation was large as indicated by a large coefficient of determination although the difference was also obvious in the case of a small window size (e.g. 3 times the average data spacing). This might be caused by the different distributions of data points in two subset point clouds of the same source (e.g. TLS-subset1 and TLS-subset2). When the TLS-subset1 was compared to the Photo-subset1, the correlation was reduced especially when the window sizes used were small. As expected, a larger window size could lead to a larger coefficient of determination. This is because greater spatial variation existing in a larger window size reduced the effects of the measurement errors and the varying distributions of individual data points. It is also interesting to witness that the improvement in R2 using a point cloud of a higher density was relatively small for the cases investigated. 


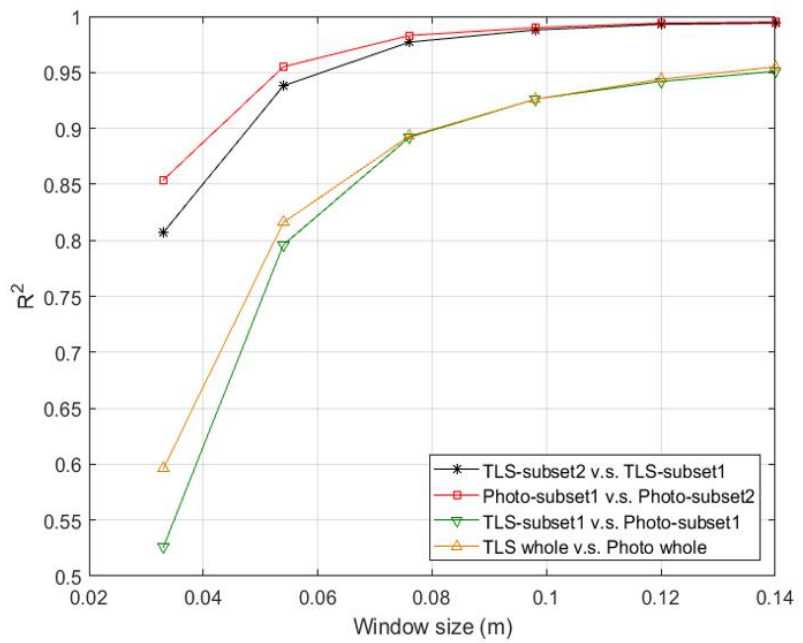

(a)

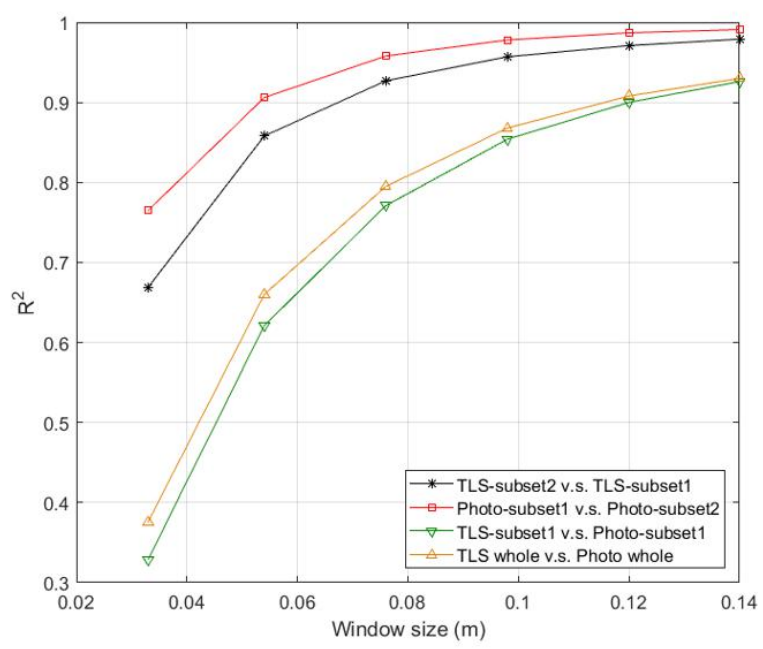

(b)

Figure 6. The coefficient of determination $\mathrm{R} 2$ for the correlation between the surface roughness maps derived using SfM and TLS, respectively: (a) RMSH, (b) standard deviation of slope.

In this case study, the R2 was reduced significantly at smaller window sizes partly because the terrain surface considered had a very small spatial variation in those small window size(s). If the spatial variation in a window size was large (e.g. the window size used was large enough such as the 13 times average data spacing in this study, or the spatial variation in a small window was adequately large), the effect of measurement errors on R2 would be relatively small.

\section{CONCLUSIONS}

It was found that the measurement accuracy of the SfM technique can be close to that of TLS if appropriate quality settings were used for generating point clouds from suitable images. For the surface roughness descriptors and the terrain surface considered, the correlations between surface roughness maps derived using SfM and TLS were relatively small when the window size used was relatively small, likely due to the different characteristics of measurement errors of those two techniques and the small spatial variations in elevations of the terrain surface. Although the small correlation could be improved by using a higher data density, the improvement was small. A larger correlation was observed when a larger window size was used because the effect of the differences in measurement errors for the case of a stronger spatial variation signal (achieved through a larger window) was reduced.

\section{ACKNOWLEDGEMENTS}

The authors are grateful for the financial support from Xi'an Jiaotong-Liverpool University or XJTLU (RDF-18-01-40), Key Program Special Fund in XJTLU (KSF-E-40) and Natural Science Foundation of Jiangsu Province (Grant number BK20160393). Appreciation also goes to the student Yan Li, who assisted with the analyses for the precision of the SfMderived point clouds at different quality settings.

\section{REFERENCES}

Aguilar, F. J., Agüera, F., Aguilar, M.A., Carvajal F., 2005. Effects of terrain morphology, sampling density, and interpolation methods on grid DEM accuracy. Photogrammetric Engineering \& Remote Sensing, 71(7), 805-816.

Ajayi, O.G., Salubi, A.A., Angbas A.F., Odigure, M.G., 2017. Generation of accurate digital elevation models from UAV acquired low percentage overlapping images. International Journal of Remote Sensing, 38(8-10), 3113-3134.

Brubaker, K.M., Myers, W.L., Drohan, P.J., Miller D.A., Boyer, E.W., 2013. The Use of LiDAR Terrain Data in Characterizing Surface Roughness and Microtopography. Applied and Environmental Soil Science, vol. 2013, 13 pages.

Brunori, C.A., et al., 2013. Characterization of active fault scarps from LiDAR data: a case study from Central Apennines (Italy). International Journal of Geographical Information Science, 27(7), 1405-1416.

Chen, Y.M., Zhou, Q.M., 2013. A scale-adaptive DEM for multi-scale terrain analysis. International Journal of Geographical Information Science, 27(7), 1329-1348.

Colomina, P.M., 2014. Unmanned aerial systems for photogrammetry and remote sensing: A review. ISPRS Journal of Photogrammetry and Remote Sensing, 92, 79-97.

Fan L., Smethurst, J., Atkinson, P., Powrie, W., 2014. Propagation of vertical and horizontal source data errors into a TIN with linear interpolation, International Journal of Geographical Information Science, 28(7), 1378-1400.

Fan L., Atkinson P.M., 2015. Accuracy of Digital Elevation Models Derived From Terrestrial Laser Scanning Data. IEEE Geoscience and Remote Sensing Letters, 12(9), 1923-1927.

Fan L., Atkinson P.M., 2018. A new multi-resolution based method for estimating local surface roughness from point clouds. ISPRS Journal of Photogrammetry and Remote Sensing, 144, 369-378.

Grohmann, C. H., Smith, M. J., Riccomini, C., 2011. Multiscale analysis of topographic surface roughness in the Midland Valley, Scotland. IEEE Transactions on Geoscience and Remote Sensing, 49(4), 1200-1213.

Hugenholtz, C.H. Brown O.W., Barchyn, T.E., 2013. Estimating aerodynamic roughness $(\mathrm{z} 0)$ from terrestrial laser scanning 
point cloud data over un-vegetated surfaces. Aeolian Research, $10,161-169$.

Kraus, K., Karel, W., Briese C., Mandlburger, G.,2006. Local accuracy measures for digital terrain models. The Photogrammetric Record, 21(116), 342-354.

Milenković, M., Pfeifer N., Glira, P., 2015. Applying Terrestrial Laser Scanning for Soil Surface Roughness Assessment. Remote Sensing, 7, 2007-2045.

Nield J. M., Wiggs, G. F. S., 2011. The application of terrestrial laser scanning to aeolian saltation cloud measurement and its response to changing surface moisture. Earth Surf. Processes Landforms, 36(2), 273-278.

Prosdocimi, M., Calligaro, S., Sofia, G., Dalla Fontana, G., Tarolli, P., 2015. Bank erosion in agricultural drainage networks: new challenges from structure-from-motion photogrammetry for post-event analysis. Earth Surf. Process. Landforms, 40, 1891-1906.

Ruiz, J. J., Diaz-Mas, L., Perez, F., Viguria, A, 2013. Evaluating the accuracy of DEM generation algorithms from UAV imagery, International Archives of the Photogrammetry, Remote Sensing and Spatial Information Sciences, Volume XL-1/W2, 2013 UAV-g2013, 4 - 6 September 2013, Rostock, Germany.

Smith M.W., Carrivick, J.L., Quincey, D.J., 2016. Structure from motion photogrammetry in physical geography. Progress in Physical Geography, 40(2), 247-275.

Marteau, B., Vericat, D., Gibbins, C., Batalla, R. J., Green, D. R., 2017. Application of Structure-from-Motion photogrammetry to river restoration. Earth Surf. Process. Landforms, 42, 503-515.

Mancini, F., Dubbini, M., Gattelli, M., Stecchi, F., Fabbri, S., Gabbianelli, G., 2013. Using Unmanned Aerial Vehicles (UAV) for High-Resolution Reconstruction of Topography: The Structure from Motion Approach on Coastal Environments. Remote Sensing, 2013, 5(12), 6880-6898.

Westoby, M.J., Brasington, J., Glasser, N.F., Hambrey, M.J., Reynolds, J.M.,2012. 'Structure-from-Motion' photogrammetry: A low-cost, effective tool for geoscience applications, Geomorphology, 179, 300-314. 